\title{
Rickettsia parkeri colonization in Amblyomma maculatum: the role of superoxide dismutases
}

\author{
Gary Crispell, Khemraj Budachetri and Shahid Karim*
}

\begin{abstract}
Background: The Gulf Coast tick (Amblyomma maculatum) is an arthropod vector of Rickettsia parkeri, the causative agent of American boutonneuse fever and an infectious agent of public health significance. In this study, we evaluated the biological significance of the superoxide dismutases (SODs) of A. maculatum in hematophagy and $R$. parkeri colonization within the tick host.

Methods: An RNA interference approach was used to measure the functional roles of tick SODs (Cu/Zn-SOD and $M n-S O D)$ in $R$. parkeri colonization of the tick vector. Total microbial load, $R$. parkeri infection rate, and compensatory mechanisms by tick genes were examined using quantitative polymerase chain reaction (PCR) and quantitative reverse-transcriptase PCR assays. SOD enzymatic activity assays and malondialdehyde (MDA) lipid peroxidation were employed to determine the redox states in the tick tissues.

Results: Knockdown of the Cu/Zn-SOD gene caused the upregulation of Mn-SOD in transcript levels. Single and dual knockdowns of the SOD genes caused an increase in MDA lipid peroxidation while SOD enzymatic activities did not show a significant change. Mn-SOD knockdown resulted in a substantial increase in the microbial load; however, CU/Zn-SOD transcript depletion prompted an upsurge in the midgut bacterial load, and significantly decreased the bacterial load in salivary gland tissues. Additionally, Cu/Zn-SOD transcript silencing led to significantly fewer R. parkeri DNA copy numbers in both tick tissues (midguts and salivary glands).

Conclusions: SOD enzymes play an important function in the regulation of bacterial communities associated with tick vectors and also in the defense mechanisms against the damage caused by reactive oxygen species within the tick. Knockdown experiments increased the levels of total oxidative stress in ticks, revealing the interplay between SOD isozymes that results in the transcriptional regulation of tick antioxidants. Moreover, the tick's Cu/Zn-SOD aids in the colonization of $R$. parkeri in tick tissues providing evidence of A. maculatum's vectorial success for a spotted fever group rickettsial pathogen.
\end{abstract}

Keywords: Amblyomma maculatum, Rickettsia parkeri, Tick, American boutonneuse fever, Superoxide dismutase, Reactive oxygen species, Selenoprotein, Lipid peroxidation, Bacterial load

\footnotetext{
* Correspondence: Shahid.Karim@usm.edu

Department of Biological Sciences, University of Southern Mississippi,

Hattiesburg, MS 39406, USA
} 


\section{Background}

The Gulf Coast tick, Amblyomma maculatum, is a recently emerged arthropod that is posing an increasingly significant risk to public health [1]. This tick covers a geographical range encompassing the coastal areas of the Eastern Atlantic and the Gulf of Mexico within the United States. It is an arthropod vector of the spotted fever group rickettsial pathogen, Rickettsia parkeri, which causes American boutonneuse fever, a similar but milder form of Rocky Mountain spotted fever. Vector competence in ticks (pathogen acquisition, colonization, and transmission) is a multifactorial process involving several genes and numerous gene networks within the tick organs (midgut and salivary glands).

Tick blood meal digestion elevates the reactive oxygen species (ROS) level, which in turn can severely harm cellular components, thereby promoting apoptosis. Hydroxyl radicals, superoxide anions $\left(\mathrm{O}^{2-}\right)$, and hydrogen peroxide $\left(\mathrm{H}_{2} \mathrm{O}_{2}\right)$ are generated through ROS. The manganese superoxide dismutase (Mn-SOD) enzyme catalyzes a small percentage of $\mathrm{O}^{2-}$ to $\mathrm{H}_{2} \mathrm{O}_{2}$, as molecular oxygen is consecutively reduced to $\mathrm{H}_{2} \mathrm{O}$ by the electron transport chain complexes. Several other mitochondrial enzymes facilitate the additional reduction of $\mathrm{H}_{2} \mathrm{O}_{2}$ into $\mathrm{H}_{2} \mathrm{O}$ and molecular oxygen. Under normal circumstances, ROS partake in cell signaling by the intervention of select thiol residues in proteins, which have, among other capacities, a plausible role in regulating significant changes in transcriptional expression [2]. However, certain conditions can disturb the balance between the antioxidant capacity of the cell and an increase in the level of ROS. This condition, known as oxidative stress, causes permanent harm to macromolecules (proteins, lipids and DNA) and can eventually lead to cellular necrosis and cell death. Ticks must maintain homeostasis to survive, and they take blood meals of spectacular size, up to 100 times their unfed mass. Ticks must somehow avert the detrimental effects while promoting the beneficial aspects of ROS, and this suggests that there are specific factors for maintaining homeostasis within the tick itself and possibly at the tick-host interface. To achieve redox homeostasis in response to the detrimental effects of ROS, the tick antioxidant system acts through enzymes, such as catalase (Cat), superoxide dismutases (SODs), glutathione peroxide, glutathione-S-transferase, glutathione reductase (GSHR), selenoenzymes, and non-enzymatic molecules [3-6].

Localized ROS generation has been described in arthropods as the first line of defense against infectious agents [7, 8]. Tick-borne pathogens manipulate the gene expression of their host vector to ensure their survival, replication, and transmission to the mammalian host. Evidence of a well-organized tick antioxidant system lends support to the idea that tick-borne pathogens manipulate the system so that it is beneficial for their survival and colonization within the tick before inoculation into the mammalian host. While the role of ROS and antioxidants in the vectorial competence of ticks has not yet been explained, the redox balance and the growth and survival of $R$. parkeri in the tick host is an enigma. Hence, in this study, the biological implications of SODs in $R$. parkeri colonization within the $A$. maculatum vector are examined.

\section{Methods}

\section{Ethics statement}

All animal experiments were conducted in strict accordance with the recommendations in the Guide for the Care and Use of Laboratory Animals of the National Institutes of Health, USA. The protocol for tick blood feeding on sheep was approved by the Institutional Animal Care and Use Committee of the University of Southern Mississippi (protocol \#15101501 and \#15011402). All efforts were made to minimize animal suffering.

\section{Ticks and other animals}

Gulf Coast ticks (A. maculatum) were maintained at the University of Southern Mississippi according to established methods [9]. Unfed adult ticks were obtained from Oklahoma State University's Tick Rearing Facility (Stillwater, OK, USA). Rickettsia parkeri-infected A. maculatum ticks were maintained in the laboratory after collection from the field [10]. Adult ticks were kept at room temperature with approximately $90 \%$ relative humidity under a photoperiod of $14 \mathrm{~h}$ light/10 h dark before infestation on sheep. Ticks were blood-fed on sheep and were either allowed to feed to repletion or were removed at 3-10 days after attachment, depending on the experimental protocol. Adult ticks were fed on sheep and immature ticks were fed on hamsters (specifically used for this study), and the animal studies were performed in accordance with protocols approved by the Institutional Animal Care and Use Committee (IACUC) at the University of Southern Mississippi.

\section{Bioinformatics analyses}

The coding sequences of SODs for A. maculatum genes used in this study were obtained from an A. maculatum sialotranscriptome study [3]. Nucleotide sequences were conceptually translated and initially aligned using ClustalX2 [11, 12] and graphically presented using Jalview [13]. Phylogenetic relationships were inferred by MEGA 6 [14] using maximum likelihood method using JTT matrix-based model [15]. 
Tick midgut and salivary gland preparation

The unfed and partially blood-fed female adult ticks were dissected within $4 \mathrm{~h}$ of removal from the sheep. The remarkable contribution of midgut and salivary gland tissues in tick's hematophagy, and potential role of these tissues in pathogen colonization, propagation, and saliva-assisted transmission to the vertebrate host led us to focus on these tissues in this study [16]. The tick midgut and salivary gland tissues were dissected in ice-cold M-199 buffer as described by Morgan et al. [17, 18]. After removal, the midguts and salivary glands were washed gently in the same ice-cold buffer. The tick tissues were either stored immediately after dissection in RNAlater (Invitrogen, Carlsbad, CA, USA) prior to mRNA extraction, or in protein extraction buffer.

\section{RNA preparation, CDNA synthesis, and quantitative reverse-transcriptase (qRT)-PCR}

Extraction of total RNA, cDNA synthesis, and qRT-PCR were conducted as previously described [19]. Briefly, the tick midguts and salivary gland tissues stored in RNAlater were used for total RNA extraction using an Illustra $^{\text {Tx }}$ RNAspin Mini Isolation kit (GE Healthcare, Piscataway, NJ, USA) according to the manufacturer's instructions. The RNA concentration was determined using a Nanodrop spectrophotometer (Thermo Fisher Scientific, Wilmington, DE, USA). Total RNA $(1 \mu \mathrm{g})$ was reverse transcribed into cDNA using iScript cDNA synthesis kit (Bio-Rad Inc., Hercules, CA, USA) according to the manufacturer's instructions . The gene-specific primer sequences designed to amplify specific cDNA fragments from $A$. maculatum tissues are listed in the Table. Transcriptional gene expression of the SODs in uninfected (naïve) ticks was normalized against the $\beta$-actin gene, while glyceraldehyde 3-phosphate dehydrogenase $(G A P D H)$ was used to normalize gene expression in $R$. parkeri-infected tick tissues. The selection of $\beta$-actin and GAPDH reference genes was based on their stable gene expression levels in uninfected and rickettsialinfected ticks [19]. All genes used in this study were first amplified using gene-specific primers (Table 1), and their sequences were confirmed by sequencing prior to dsRNA synthesis or gene expression studies. First-strand cDNA was used to measure mRNA levels using qRT-PCR. SYBR Green qPCR Master Mix (Bio-Rad Inc., Hercules, CA, USA), $25 \mathrm{ng}$ of cDNA, and $150 \mathrm{nM}$ of gene-specific primers were used in each reaction mixture [6]. The qRTPCR mixtures were subjected to $10 \mathrm{~min}$ at $95^{\circ} \mathrm{C}$, followed by 35 cycles of $15 \mathrm{~s}$ at $95^{\circ} \mathrm{C}, 30 \mathrm{~s}$ at $60^{\circ} \mathrm{C}$, and $30 \mathrm{~s}$ at $72^{\circ}$ $\mathrm{C}$ using the CFX96 Real Time System (Bio-Rad Inc.).

\section{Double-stranded RNA (dsRNA) synthesis, tick injections, and hematophagy}

Synthesis of dsRNA for SOD analysis and tick manipulations was performed according to the methods described previously [20, 21]. Briefly, PCR product, purified using a QIAquick PCR purification kit (Qiagen, Valencia, CA, USA), was used in a secondary PCR using the same primers with SOD-specific sequences (Table 1), but with the addition of flanking T7 sequences that allow for the binding of reverse transcriptase and the generation of dsRNA. After confirming the T7-flanked SOD gene sequence, the secondary PCR product was reverse transcribed

Table 1 Gene-specific quantitative reverse-transcriptase PCR primers used in the experiments

\begin{tabular}{|c|c|c|c|c|}
\hline Gene & Accession number & Forward primer 5'-3' & Reverse primer 5'-3' & Size (bp) \\
\hline $\operatorname{Tr} \times R$ & J0843723 & TGTGACTACACCAACGTGCCTACA & AGTAGCCTGCATCCGTTCCTCTTT & 175 \\
\hline Catalase & J0843741 & AAAGGACGTCGACATGTTCTGGGA & ACTTGCAGTAGACTGCCTCGTTGT & 173 \\
\hline GSHR & J0844062 & ACCTGACCAAGAGCAACGTTGAGA & ATCGCTTGTGATGCCAAACTCTGC & 170 \\
\hline MnSOD (SOD3) & JO843979 & GCATCTACTGGACAAACCTCTC & GCAGACATCAGGCCTITGA & 115 \\
\hline CU/ZnSOD (SOD1) & JO844140 & GGAACCGAAGACAGCAAGAA & GAGAAGAGGCCGATGACAAA & 143 \\
\hline Duox & N/A & ATGACGCACAGCCTGTATATT & TGTCCAGAGTGAAGACGATTG & 123 \\
\hline $16 S$ rRNA & N/A & AGAGTTGATCCTGGCTCAG & CATGCTGCCTCCCGTAGGAGT & N/A \\
\hline Actin & JO842238 & TGGCTCCTTCCACCATGAAGATCA & TAGAAGCACTTGCGGTGCACAATG & 169 \\
\hline GAPDH & JO842341 & CACCCATCACAAACATGGGTGCAT & TTCAGGAAATGAAGCCTGCCAGC & 175 \\
\hline OmpB & AF123717 & CAAATGTTGCAGTTCCTCTAAATG & AAAACAAACCGTTAAAACTACCG & 96 \\
\hline \multicolumn{5}{|c|}{ Probe: 6-FAM-CGCGAAATTAATACCCTTATGAGCAGCAGTCGCG-BHQ-1 } \\
\hline SelM & J0842653 & ATGATACCTGAATGGCCATCCGCA & TGATCGCGGGTCATCTTCTCCAAA & 171 \\
\hline eEFSec (SEF) & KC989559 & TGGCTCCAGAAATGCTGCTCATTG & ACGCCTTTGCGACTCTTCTCCTTA & 157 \\
\hline Sels & J0842687 & AGAACAAGTGCACCACAACAGCAG & ATTTCTTGCATCCTTCGACGTGCC & 107 \\
\hline SelN & KC989560 & TTAGTTTGGACACTGTGGACGGGT & AGGCTTCTCTAACAACGGCACTCA & 150 \\
\hline SelK & J0843326 & AGTTCCAGCAGGTCATCAGTGTCA & TCCAGGAATAGGGCAGTCCATTGT & 132 \\
\hline Salp25D & J0843645 & TGCCGCGCTGTCTTTATTATTGGC & AGTTGCACGGAGAACCTCATCGAA & 102 \\
\hline
\end{tabular}


into RNA using a T7 Quick High Yield RNA synthesis kit (New England Biolabs, Ipswich, MA, USA) by incubating the PCR product with T7 polymerase overnight at $37^{\circ} \mathrm{C}$. The resulting dsRNA was purified by ethanol precipitation and its concentration measured spectrophotometrically using a Nanodrop device (Thermo Fisher Scientific). The product was visualized by gel electrophoresis using a $2 \%$ agarose gel. The dsRNAs (dsMn-SOD and dsCu/Zn-SOD) were diluted to working concentrations of $1 \mu \mathrm{g} / \mu \mathrm{l}$. The same protocol was used to synthesize dsRNA-LacZ to be used as a dsRNA control. Forty-five unfed adult female ticks (uninfected or $R$. parkeri-infected) were microinjected with $1 \mu \mathrm{l}$ of dsRNA-SOD or dsLacZ using a 27-gauge needle, after which they were kept overnight at $37^{\circ} \mathrm{C}$ to alleviate needle trauma and promote survival. The surviving ticks were used to infest a sheep and allowed to blood feed in the presence of male ticks. For sample collection, 10 partially fed experimental control ticks were removed on days 5 and 7 post-tick infestation, and the remaining ticks were allowed to remain attached and blood feed until repletion. The feeding success of the individual ticks was evaluated by recording their attachment duration, repletion weight, and the ability to oviposition [22]. Partially fed ticks removed from the dsLacZ or dsSOD groups were dissected to obtain their midguts and salivary gland tissues.

\section{Quantification of total bacterial load}

The bacterial load in each tick tissue was estimated as described previously [6, 23]. Briefly, $25 \mathrm{ng}$ of cDNA from the tick tissues, $200 \mu \mathrm{M}$ 16sRNA gene primers, and $2 \times$ iTaq Universal SYBR green mix (BioRad Inc.) in a $25-\mu$ l volume reaction was used with the following thermocycler parameters: $94{ }^{\circ} \mathrm{C}$ for 5 min followed by 35 cycles at $94{ }^{\circ} \mathrm{C}$ for $30 \mathrm{~s}, 60{ }^{\circ} \mathrm{C}$ for $30 \mathrm{~s}$ and $72{ }^{\circ} \mathrm{C}$ for $30 \mathrm{~s}$. Standard curves were used to estimate the copy numbers of each gene. The bacterial copy numbers were normalized against $A$. maculatum actin copy numbers in the uninfected ticks and GAPDH in the R. parkeri-infected ticks. All samples were run in triplicate.

\section{Quantification of R. parkeri copy numbers in tick tissues}

The level of infection with $R$. parkeri within the tick tissues (midguts and salivary glands) was quantified using a probe-based qPCR method described previously [10]. Briefly, 0.4 $\mu \mathrm{M}$ of probe, $0.7 \mu \mathrm{M}$ of each forward and reverse primer (Rpa129F, Rpa224R) (Table 1) and $8 \mathrm{mM}$ of $\mathrm{MgSO}_{4}$ reaction mixture was subjected to one cycle each of $50{ }^{\circ} \mathrm{C}$ for $2 \mathrm{~min}$ and $95{ }^{\circ} \mathrm{C}$ for $2 \mathrm{~min}$, then 45 cycles of $95{ }^{\circ} \mathrm{C}$ for $15 \mathrm{~s}$ and $60^{\circ} \mathrm{C}$ for $30 \mathrm{~s}$. A standard curve was used to calculate the $R$. parkeri copy numbers in the tick samples. All samples were run in triplicate.

\section{Quantification of total oxidative stress levels}

A malondialdehyde (MDA) lipid peroxidation assay was used to quantify the total oxidative stress levels in the tick tissues. Degradation of lipids as a result of oxidative damage was estimated by quantification of MDA through the use of a Lipid Peroxidation MDA Assay Kit (Sigma-Aldrich, St. Louis, MO, USA) following the manufacturer provided protocol. Twenty milligrams of midgut tissue from individual ticks was filtered through a $0.2 \mu \mathrm{m}$ filter before the assay was performed according to the manufacturer's instructions.

\section{Quantification of SOD activity}

Superoxide enzymatic activity was quantified in individual midgut and salivary gland tissues using the Superoxide Dismutase Assay Kit (Cayman Chemical Co, Ann Arbor, MI, USA). The tissues were processed according to the manufacturer's instructions.

\section{Data analysis}

All data are expressed as mean \pm SEM unless otherwise stated. Statistical significance between the two experimental groups or their respective controls was determined by the $t$-test). Comparative differences amongst the multiple experimental groups were determined by analysis of variance with statistically significant $P$-values of $<0.05$ (Graphpad Prism 6.05, La Jolla, CA, USA). Transcriptional expression levels were determined using Bio-Rad software (Bio-Rad CFX MANAGER v.3.1), and the expression values were considered significant if the $P$-value was 0.05 when compared with the control.

\section{Results}

\section{Bioinformatic analyses}

In this study, the functional role of A. maculatum superoxide dismustases (SODs) in hematophagy, and bacterial colonization of endosymbionts and pathogenic microbe $R$. parkeri was determined. Two gene sequences of tick superoxide dismutases cytosolic with signal peptide $(\mathrm{Cu} / \mathrm{ZnSOD})$ and intramitrocondrial scavenger ( $\mathrm{Mn}$ $S O D$ ) were selected to analyze for the presence of secretory signal peptide using SignalP [24] server (http://www.cbs.dtu.dk/services/SignalP/). The amino acid residues deduced from the transcripts of two SODs identified earlier [3] were translated and visualized using Jalview and observed six histidine residues in each sequences showing metal-binding sites (Additional file 1: Figure S2A, B). The four histidine imidazoles are coordinated to $\mathrm{Cu}(\mathrm{II})$ while two other and including one common histidine imadazoles binds to $\mathrm{Zn}$ (II) metal in $\mathrm{Cu} / \mathrm{Zn}$-SOD dismutase [25]. The Mn-SOD localized in mitochondria in eukaryotes, and found in cytoplasm in prokaryotes shares common structural properties with Fe-SOD [26]. The phylogenetic tree was built in MEGA software version 6 [14] to provide evolutionary 
significance between mitochondrial and cytoplasmic superoxide dismutases (Additional file 1: Figure S1). Phylogenetically $\mathrm{Mn}$-SOD and $\mathrm{Cu} / \mathrm{Zn}$-SOD fit in two different clades, sharing common node with corresponding SODs from another Amblyomma ticks (Additional file 1: Figure S1).

\section{Time-dependent transcriptional gene expression analysis}

The time-dependent transcriptional expression of the $\mathrm{Cu} / \mathrm{Zn}$-SOD and $\mathrm{Mn}$-SOD genes was examined in unfed and partially blood-fed tick tissues (midguts and salivary glands) (Fig. 1). The time-dependent expression of the $\mathrm{Cu} / \mathrm{Zn}-\mathrm{SOD}$ gene in the tick midgut tissues showed its highest transcript level during the unfed phase followed by significant depletion after attachment, and the transcript level remained down-regulated as the tick blood feeding switched from day 2 to day 8 (Fig. 1a). Interestingly, the transcript level of the $\mathrm{Cu} / \mathrm{Zn}-\mathrm{SOD}$ gene in the tick salivary glands was up-regulated during the slow and fasting feeding phases of tick blood feeding, signifying a potential functional role in tick hematophagy (Fig. 1a). By contrast, the transcript level of the $M n-S O D$ gene in the tick midgut tissues upon blood feeding was up-regulated and remained 2-fold higher than in the unfed tissues in the 2- and 6 day-fed ticks (Fig. 1b); however, the salivary glands showed a 2- to 6-fold increase in the transcript level on days 4 and 6 post-attachment to the vertebrate host (Fig. 1b). Surprisingly, the transcript level of $M n-S O D$ decreased in the fast-feeding phase (8 days post-infestation), implying compensation by $\mathrm{Cu} / \mathrm{Zn}-\mathrm{SOD}$ or a reduced biological need in the cell.

\section{Impact of single and multiple SOD knockdowns in uninfected ticks}

Transcriptional gene expression: To investigate the functional role(s) of the secreted extracellular and mitochondrial SOD enzymes, genes were disrupted using RNA interference, and the effects of the single and dual knockdowns upon the transcriptional gene expression of Cat, dual oxidase (Duox), GSHR and thioredoxin reductase, as well as oxidative stress, bacterial load, and enzymatic activity was investigated (Figs. 2, 3 and 4). A Cu/Zn$S O D$ gene silencing efficiency of 77 and $76 \%$ was achieved in 5 dpi tick midgut and salivary gland tissues respectively (Fig. 2a). On the contrary, $M n-S O D$ transcript silencing efficiency of 76 and $94 \%$ was obtained in tick midgut and salivary tissues respectively (Fig. 2b). Intriguingly, simultaneous gene silencing of $\mathrm{Cu} / \mathrm{Zn}-\mathrm{SOD}$ and $\mathrm{Mn}-\mathrm{SOD}$ showed 90 and $98 \%$ silencing efficiency of $\mathrm{Cu} / \mathrm{Zn}$-SOD verses 63 and $71 \%$ of $M n-S O D$ in midgut and salivary gland tissues respectively (Fig. 2c). Regardless of the single $(\mathrm{Cu} / \mathrm{Zn}-\mathrm{SOD}$ or $\mathrm{Mn}-\mathrm{SOD})$ and dual $(\mathrm{Cu} / \mathrm{Zn}-\mathrm{SOD}$ and $M n-S O D)$ knockdowns, there were no significant changes in tick behavior or phenotype in terms of the tick engorgement weight, the attachment duration (Additional file 1: Figure S3). Surprisingly, knockdown of tick SODs did not translate into the depletion of SOD enzymatic activity in midgut tissues but salivary glands showed a decrease in $\mathrm{Cu} / \mathrm{Zn}-\mathrm{SOD}$ and dual knockdown tissues, dsMn-SOD $(P=0.002)$, and dual SOD injected $(P=0.036)$ (oneway ANOVA using multiple comparison with Tukey test) (Fig. 3a, b). The lack of correlation between $\mathrm{Cu} /$ $\mathrm{Zn}$-SOD level may be due to the residual amount of proteins already present in the tissues. $\mathrm{Cu} / \mathrm{Zn}-\mathrm{SOD}$ silencing in the partially blood-fed tick tissues upregulated $M n-S O D$ gene expression; however, Cat, Duox, and GSHR gene expression in the salivary glands remained unchanged (Fig. 2a). By contrast, $M n-S O D$ silencing in uninfected (naïve) ticks showed a 4-35-fold increase in $\mathrm{Cu} / \mathrm{Zn}-\mathrm{SOD}$ expression and, intriguingly, Duox expression in the salivary glands was significantly down-regulated $(P<0.05)$, suggesting an interplay between Duox and $M n-S O D$ in the salivary glands (Fig. 2b). Simultaneous silencing of $\mathrm{Cu} / \mathrm{Zn}$ $S O D$ and $M n-S O D$ in naïve ticks only impacted the
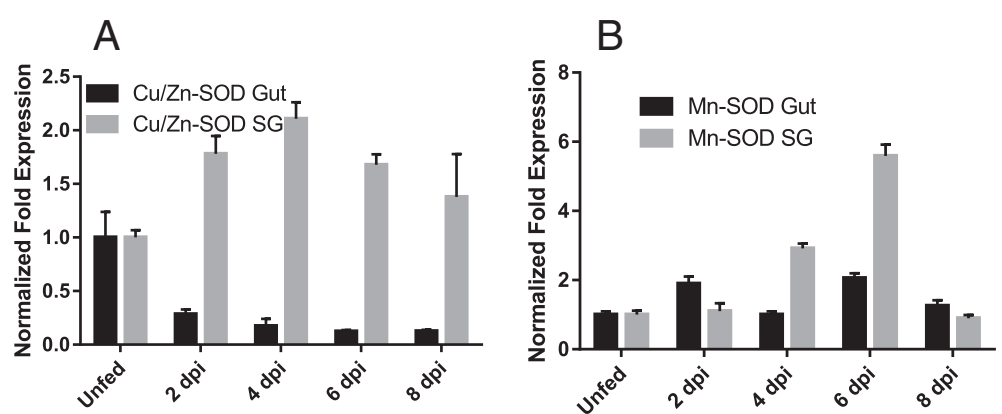

Fig. 1 Time-dependent CU/Zn-SOD and Mn-SOD transcriptional expression levels in uninfected (naïve) tick midgut and salivary gland tissues during the blood meal. The relative transcript levels of the SODs during the blood meal were measured by comparing the SOD expression levels in the midgut (Gut) and salivary gland (SG) tissues of the unfed-stage ticks. The changes in transcriptional activity of (a) CU/Zn-SOD and (b) $\mathrm{Mn}-\mathrm{SOD}$ in the A. maculatum midgut and salivary gland tissues were normalized to the unfed developmental stage using $\beta$-actin as a reference gene 


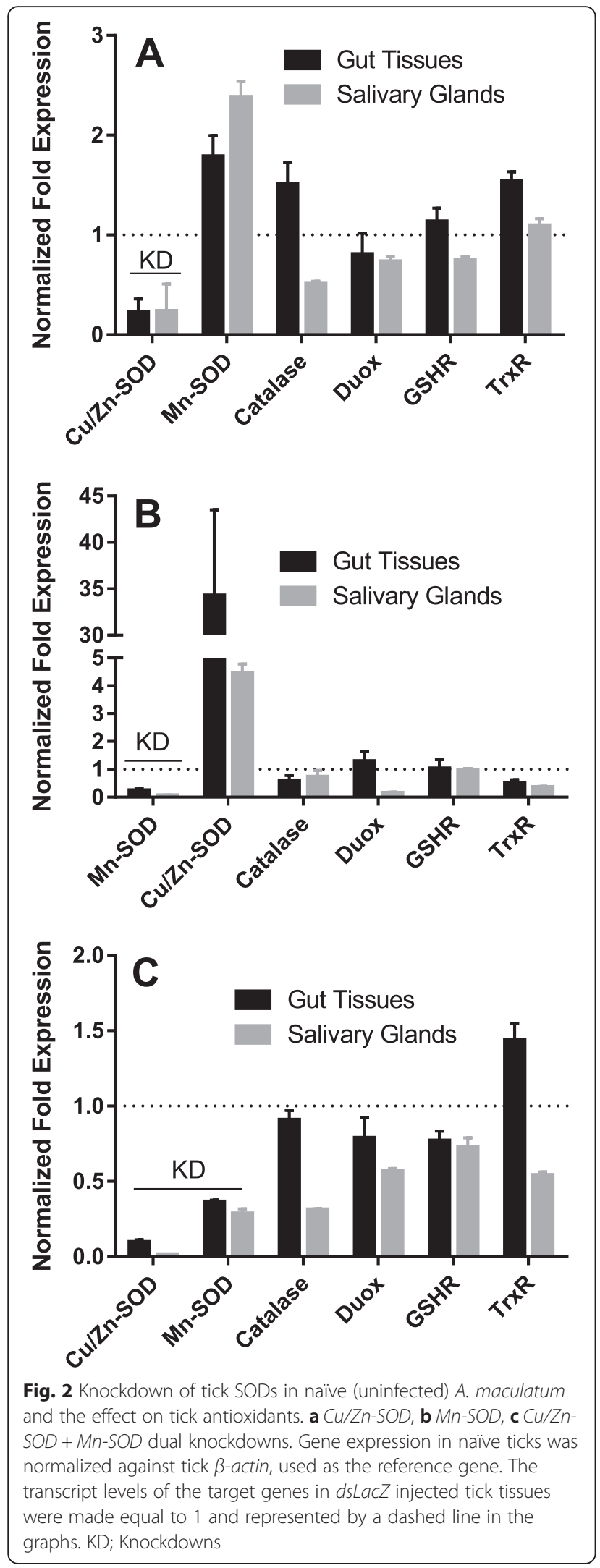

expression of selected genes in the salivary gland tissues (Fig. 2c), suggesting the tissue-specific expression of antioxidant genes during blood feeding.

Oxidative stress levels and SOD enzymatic activity: The cytosolic and mitochondrial SOD enzymatic activities were determined in the uninfected single- and simultaneously gene-silenced tick midgut and salivary gland tissues. Knockdown of $\mathrm{Cu} / \mathrm{Zn}-\mathrm{SOD}$ resulted in slightly decreased enzyme activity, while $M n-S O D$ silencing lowered the activity in the midgut and salivary gland tissue fractions compared with the control (Fig. 3a). Simultaneous silencing of $\mathrm{Cu} / \mathrm{Zn}$-SOD and $\mathrm{Mn}$-SOD produced lower $\mathrm{Cu} / \mathrm{Zn}-\mathrm{SOD}$ activity in the midgut and salivary gland tissues; while the salivary glands experienced a slight decrease in Mn-SOD activity as compared with the irrelevant dsRNA-treated tick tissues (Fig. 3b). Additionally, the level of oxidative stress, as quantified by MDA, showed a 5-6-fold increase in oxidative stress upon knockdown of $\mathrm{Cu} / \mathrm{Zn}$-SOD $(t=2.847, P=0.0465)$ and $M n-S O D(t=10, P=0.0004)$ in the midgut tissues; however, simultaneous knockdown of the expression of both genes produced only a 2-fold increase $(t=0.6904$, $P=0.529$ ) in the oxidative stress level (Fig. 3c). Quantification of the oxidative stress level in the salivary gland tissues was hampered by the insufficiently high protein concentration needed for this assay.

Native microbial load: To further understand the role of SODs in the native microbial load within the tick tissues, we calculated the overall bacterial load by quantifying the $16 S$ rRNA gene. We observed that when $M n-S O D$ was knocked down in the uninfected tick tissues the total bacterial load in the midgut and salivary gland tissues increased $\left(F_{(3,36)}=9.636, P<0.0001\right)$ (Fig. $4 \mathrm{a}$ and b). By contrast, $\mathrm{Cu} / \mathrm{Zn}-\mathrm{SOD}$ gene silencing significantly depleted the colonization of microbiota in the tick salivary glands $(t=2.794, P=0.0190)$ (Fig. 4d), suggesting a hypothetical role for this gene in the colonization of microbes in tick salivary glands before saliva-assisted transmission to the host (Fig. 4b). Intriguingly, $\mathrm{Cu} / \mathrm{Zn}$-SOD gene silencing resulted in no significant difference in the microbial load in the tick midgut tissues $(t=2.151, P=0.0570)$ (Fig. 4c). The bacterial load in tick tissues was significantly altered in dual knockdown of SODs in tick tissues $\left(F_{(3,20)}=7.493, P\right.$ $=0.0015)($ Fig. 4e, f).

\section{Impact of $\mathrm{Cu} / \mathrm{Zn}$-SOD knockdown in R. parkeri-infected ticks}

SOD expression was assessed in the tissues (midgut and salivary glands) of uninfected and $R$. parkeri-infected partially blood-fed adult ticks (Fig. 5a). Interestingly, the $\mathrm{Mn}$-SOD transcript level was up-regulated 8-fold while $\mathrm{Cu} /$ $Z n-S O D$ was down-regulated in the $R$. parkeri-infected tick midgut tissues (Fig. 5a). Rickettsia parkeri infection in the partially blood-fed salivary glands caused up-regulation of 


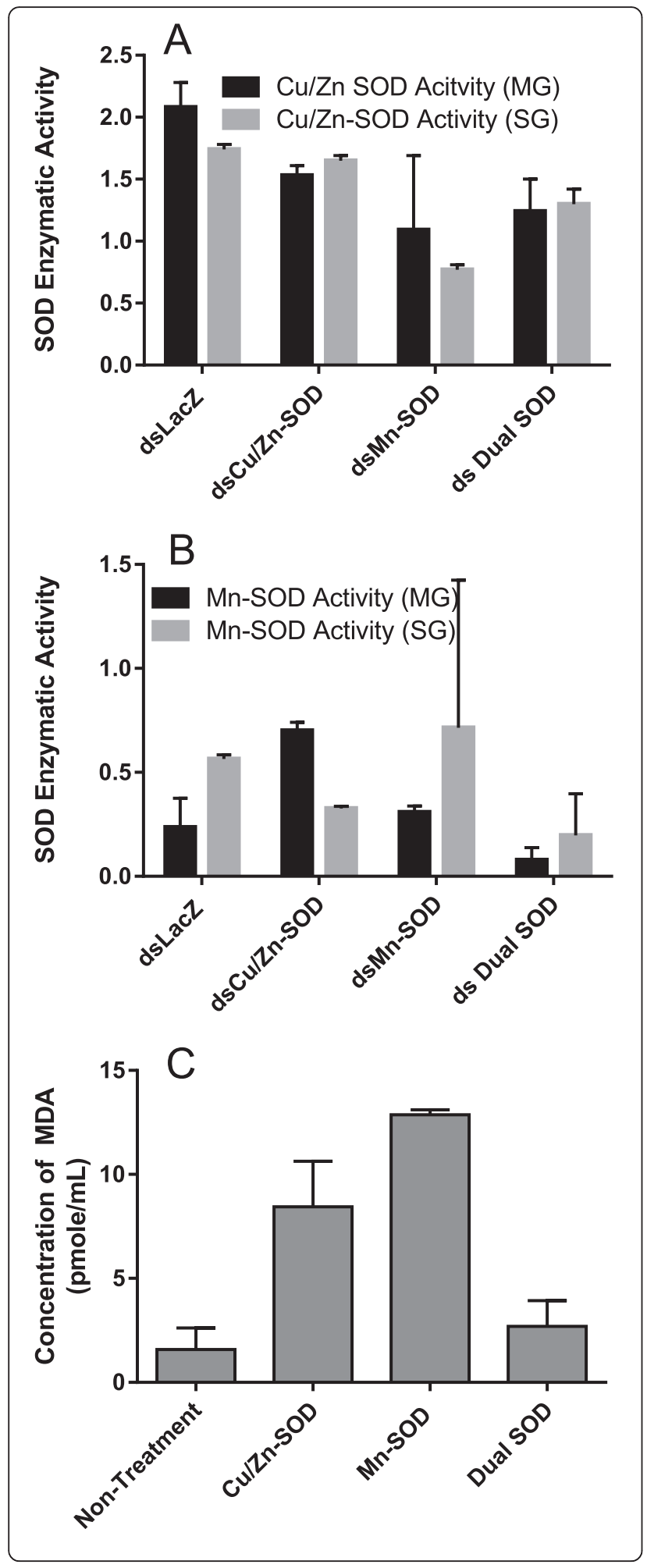

Fig. 3 Quantification of the SOD activity and total oxidative stress levels in superoxide dismutase-silenced naive tick midguts. a Cytosolic and $\mathbf{b}$ mitochondrial SOD enzymatic activities in tick midgut and salivary gland tissues were estimated using Superoxide Dismutase Assay Kit (Cayman Chemical Co, Ann Arbor, MI, USA). The tissue cytosolic fraction was used for determining the enzymatic activity of Cu/Zn-SOD, and mitochondrial fraction was reconstituted to assess Mn-SOD activity. c A Lipid Peroxidation (MDA) Assay Kit (Sigma-Aldrich, St. Louis, MO, USA) was used for estimating total oxidative stress in the tick midgut tissues. The MDA-TBA adduct was spectrometrically estimated at 532 $\mathrm{nm}$ in each control and knockdown tick midgut

the expression of both SODs $(2-8$-fold, $P<0.05)$, suggesting their potential role in pathogen colonization within the tick host (Fig. 5a). To further understand the proposed role of $\mathrm{Cu} / \mathrm{Zn}-\mathrm{SOD}$ in $\mathrm{R}$. parkeri colonization in A. maculatum, $\mathrm{Cu} / \mathrm{Zn}-\mathrm{SOD}$ was silenced using RNAi, and its effect on the native microbial load and $R$. parkeri colonization was examined (Fig. 5c, d). As anticipated, the $\mathrm{Cu} / \mathrm{Zn}$-SOD transcript in the knockdown showed 77 and $99 \%$ silencing efficiency in midgut and salivary gland tissues respectively in $5 \mathrm{dpi}$ ticks (Fig. 5b) $(P<0.05)$. Predictably, the transcriptional expression of $M n-S O D$ exhibited a 6.5-7-fold upregulation upon silencing of $\mathrm{Cu} / \mathrm{Zn}-\mathrm{SOD}$ in the partially blood-fed infected tissues (Fig. 5b). The transcript level of selected antioxidant genes (Cat, Duox, GSHR, and $\operatorname{Tr} x R$ ) decreased slightly in the $\mathrm{Cu} / \mathrm{Zn}$-SOD-silenced $R$. parkeriinfected tick tissues. Intriguingly, $\mathrm{Cu} / \mathrm{Zn}-\mathrm{SOD}$ silencing up-regulated the transcript level of selenoprotein $\mathrm{O}, \mathrm{Mn}$ $S O D$, and glutathione peroxidase (Salp25D) in the tick midguts, while $M n-S O D$ and Salp25D expression in the salivary gland tissues increased by $6-8$-fold, indicating the existence of a compensatory mechanism (Fig. 5b). Interestingly, quantification of $R$. parkeri in the gene-silenced partially blood-fed tick tissues revealed a significant decrease in the rickettsial copy numbers in midguts $(t=7.5$, $P<0.0001)$ and in salivary gland tissues $(t=8.419, P<$ 0.0001 ) (Fig. $5 \mathrm{c}$ ), suggesting a potential role for $\mathrm{Cu} / \mathrm{Zn}$ $S O D$ in $R$. parkeri colonization within the tick host. Surprisingly, the total bacterial load in $\mathrm{Cu} / \mathrm{Zn}-\mathrm{SOD}$ knockdown midgut $(t=0.5079, P=0.6225)$ and salivary gland tissues $(t=0.03569, P=0.09722)$ remained unchanged (Fig. 5d).

\section{Discussion}

Ixodid ticks are arthropods dependent on hematophagy for transition to each life stage and for maintaining their reproductive fitness. Hematophagy in ixodid tick is a phase-dependent process, starting with host attachment and establishment of the feeding site, followed by slow feeding and transition into fast feeding until repletion. At this point in the feeding process, the tick can weigh over 100 times its pre-engorgement weight as a result of the imbibed blood meal. The entire process of tick feeding is dependent on the tick's secreted salivary components that 

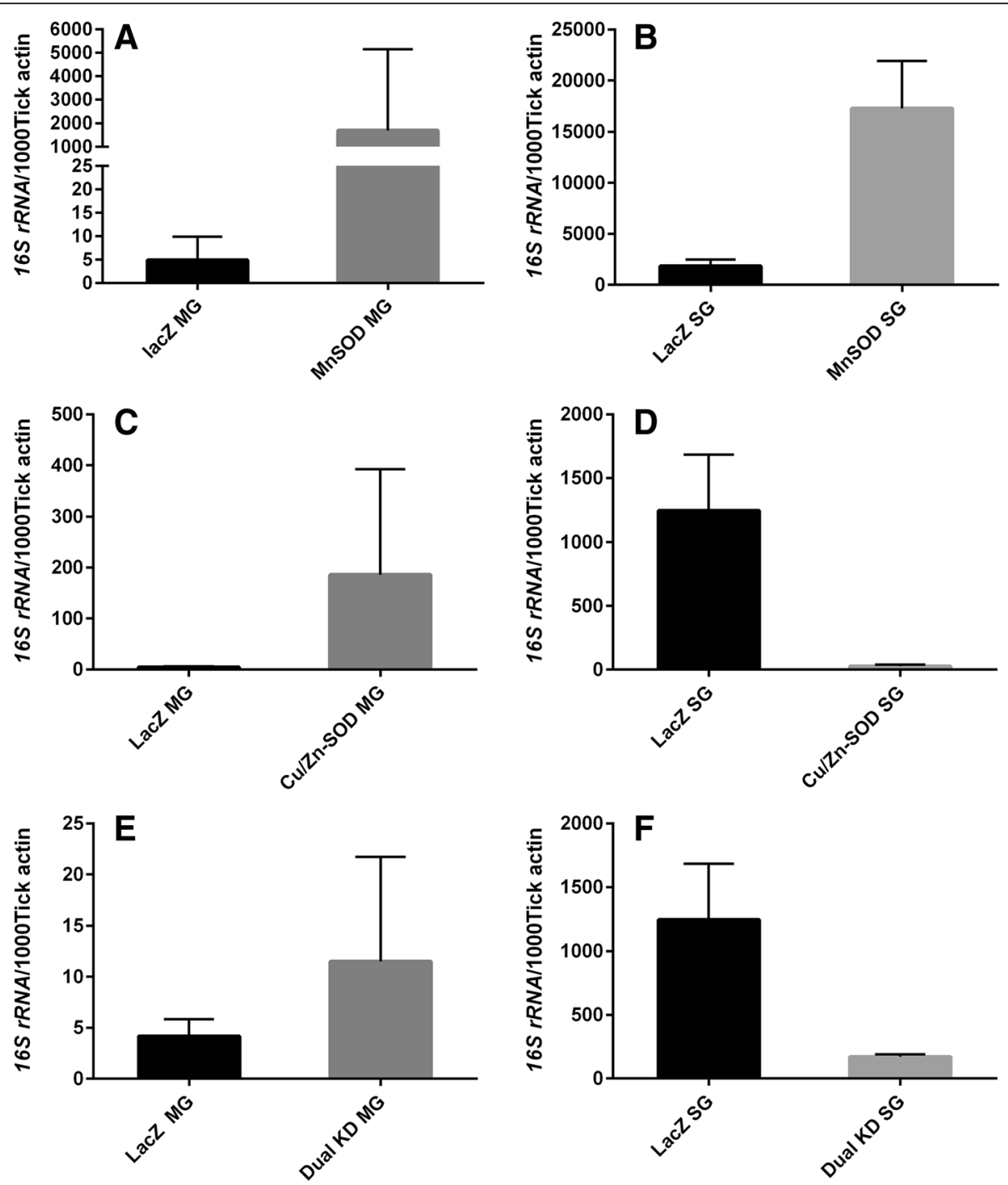

Fig. 4 Impact of SOD knockdown on microbiota load in naïve tick tissues. The bacterial load in the tick tissues (measured in copy numbers) was calculated by qRT-PCR using the bacterial $16 \mathrm{~s}$ rRNA gene and was normalized against the tick $\beta$-actin gene (copy number) in tick midguts and salivary glands each knockdown; Mn-SOD (a, b); Cu/Zn-SOD (c, d); and Dual SODs knockdown (e, f)

mechanistically facilitate successful transition of each feeding phase over the course of multiple days to weeks. Tick saliva, as shown by sialotranscriptome analysis, is composed of $\sim 5000$ putative secreted salivary proteins containing dozens of protein families [3, 27], many of which exhibit pharmacological properties capable of disarming the host's attempts to establish hemostasis or to mount a specific immune response and, ultimately, enhancing the tick's ability to blood-feed. At the same time, ticks face elevated oxidative stress both on and off the mammalian host and they must neutralize the harmful effect. The ability of ticks to counterbalance nutritional stress (starvation) and blood feeding-related stress (heme digestion) indicates the likely development of a proactive detoxification system. Complete studies of the antioxidant systems of blood-sucking arthropods are presently unavailable; nonetheless the scientific literature shows that ticks have an extensive arsenal of antioxidants and selenoproteins that allow them to survive extreme variations in redox homeostasis, with prolonged periods of starvation, the digestion of huge blood meals, and exposure to blood-related products $[4-6,20,27]$. Hence, an insight into the tick's antioxidant repertoire should offer a glimpse of its defenses against elevated levels of oxidative stress. It should also broaden our understanding of tick-pathogen interactions. Although, $A$. maculatum is a recognized arthropod vector of $R$. parkeri, this vector-pathogen pair remains the most poorly studied vector-borne disease. Here, we investigated the functional role of two tick SODs (extracellular and mitochondrial) in tick hematophagy and pathogen colonization within the 

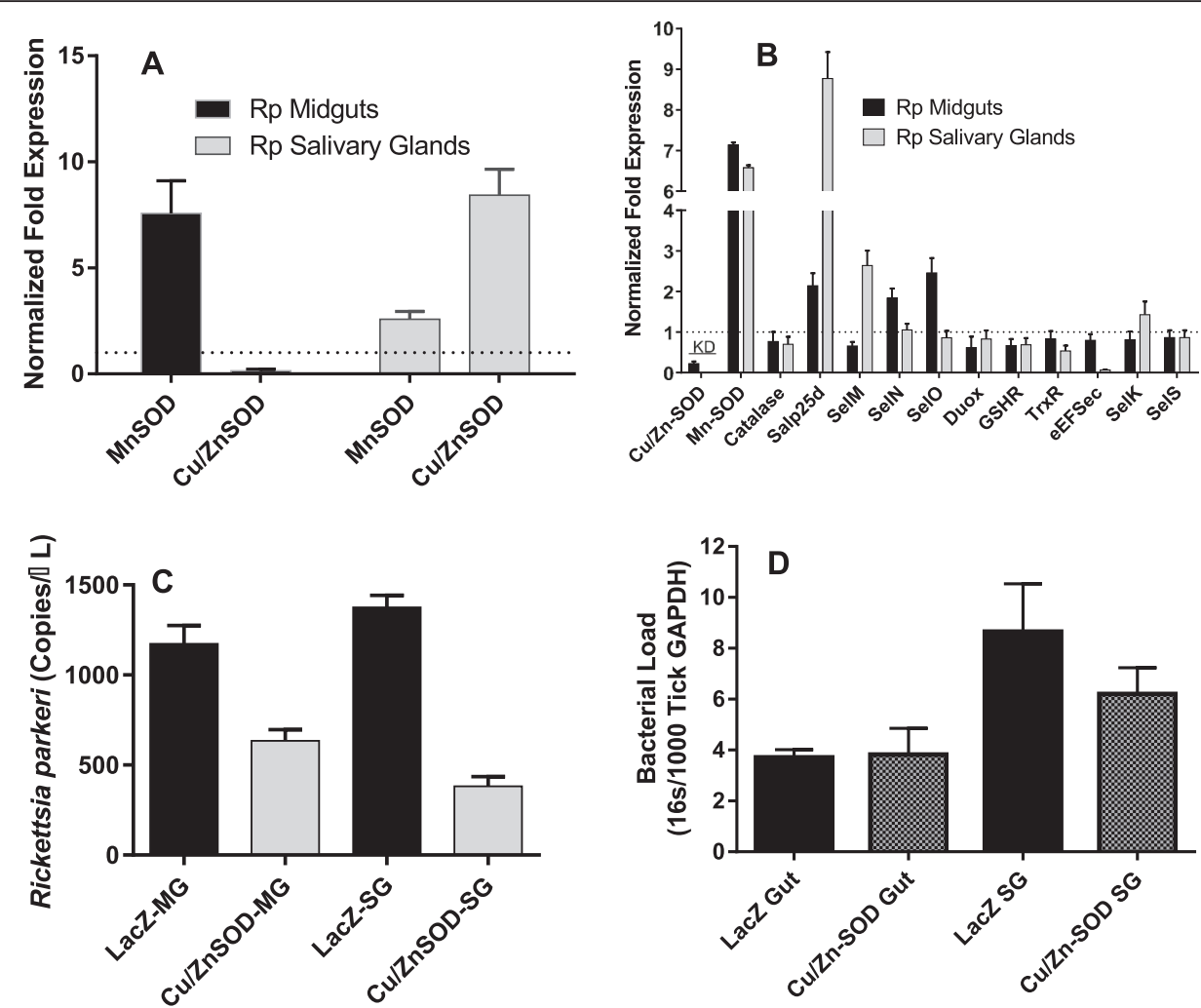

Fig. 5 Knockdown of CU/Zn-SOD in R. parkeri-infected A. maculatum. a Tissue-specific SOD transcriptional gene expression in uninfected and $R$. parkeri-infected partially blood-fed female adults tissues. A. maculatum GAPDH was used as a reference gene to normalize the gene expression data. Target gene expression in uninfected tick tissues was adjusted to 1. b Transcriptional gene expression of selected antioxidant genes in Cu/ Zn-SOD-silenced partially fed tick tissues. The transcript level of each target gene in the tissues injected with control LacZ-dsRNA was set to 1.0 as a reference point. Expression was normalized against the tick GAPDH gene. $\mathbf{c}$ R. parkeri was quantified using a real-time PCR assay designed for specific detection of the R. parkeri ompB gene in A. maculatum midgut and salivary gland in dsLacZ and dsCu/Zn-SOD injected tick tissues, and (d), the bacterial load estimation in R. parkeri-infected ticks knocked down with Cu/ZnSOD. Tick GAPDH was used as a reference gene for estimating the total bacterial load in R. parkeri-infected ticks. KD; Knockdowns

arthropod vector. Bioinformatic analysis of the $\mathrm{Cu} / \mathrm{Zn}-\mathrm{SOD}$ (cytosolic) and $\mathrm{Mn}$-SOD proteins showed amino acid sequence similarity with arthropod and vertebrate proteins (Additional file 1: Figures S1 and S2).

The two A. maculatum SODs we characterized showed quite different expression patterns. The transcriptional activity of $\mathrm{Cu} / \mathrm{Zn}-\mathrm{SOD}$ was 2 -fold up-regulated throughout the blood meal in the salivary tissues, whereas it declined in the midgut tissues upon blood meal consumption (Fig. 1a). Mn-SOD expression was up-regulated 3-6-fold in the salivary glands on days 4 and 6; however, the midgut maintained a steady expression level (Fig. 1b). Furthermore, while we obtained both single and dual gene transcriptional knockdowns, significant differences in the tick phenotype (tick engorgement weight, attachment duration, egg mass, egg conversion ratio, and hatchability) (Additional file 1: Figure S3) were lacking, a result that is similar to previous studies reporting the existence of a strong compensatory mechanism in the tick host for detoxifying superoxide and $\mathrm{H}_{2} \mathrm{O}_{2}$ [20]. The tick salivary glands undergo tremendous biochemical and physiological changes as soon as a tick attaches itself to the host, followed by engorgement and repletion [28]. In ticks, breakdown of the huge blood meal generates toxic levels of heme leading to elevated ROS production. The gut bacterial community is strongly shaped by the ROS levels upon a blood meal, resulting in a decrease in diversity and an increase in Enterobacteriaceae [6]. The establishment of vector competence (pathogen survival, colonization, and transmission) is highly specific: $R$. parkeri can only be vectored by $A$. maculatum, Ehrlichia chaffeensis by Amblyomma americanum, and Borrelia burgdorferi by Ixodes scapularis. It is postulated that these vector-borne disease agents have developed an antioxidant capacity that aids them to survive and multiply by balancing oxidative tissue-specific homeostasis. Tick-borne pathogens are vulnerable to a high level of oxidative stress; consequently, disrupting the redox metabolism offers a promising approach for the prevention of tick-borne disease agents and for disturbing tick microbiota. Our recent work 
highlighted the biological implication of thioredoxin reductase (TrxR, a selenoprotein) in preserving the natural microbiota of ticks [6], and a link between selenocysteine elongation factor and $R$. parkeri survival in the midgut tissues [4]. It has been shown that elimination of ROS is required to conserve fertility in Anopheles, Drosophila, $A$. maculatum, and mammals [20, 29-31]. To neutralize the damaging effects of ROS and attain homeostasis, tick SODs and Cat enzymes act together to catalyze the conversion of superoxide and $\mathrm{H}_{2} \mathrm{O}_{2}$. Superoxide and hydrogen peroxide facilitate the generation of the hydroxyl radical, the most reactive oxygen free radical, in the presence of iron metal $[32,33]$. Interestingly, lipid peroxidation assays showed higher total oxidative stress levels for single knockdown tick midguts, while dual knockdown revealed only a slight change in the oxidative stress level, suggesting the presence of a strong compensatory mechanism, as has been reported previously (Fig. 3c) [20]. Estimation of the total oxidative stress levels in individual tick salivary glands was not successful because of the low protein concentrations required for this assay. Intriguingly, gene knockdown of $\mathrm{Cu} / \mathrm{Zn}$-SOD or $\mathrm{Mn}$-SOD significantly increased the total bacterial load in the midgut tissues (Fig. 4a, c), whereas $\mathrm{Cu} / \mathrm{Zn}-\mathrm{SOD}$ knockdown in the salivary glands significantly reduced the total bacterial load (Fig. 4d). The total bacterial load in the dual SOD knockdown remained at a decreased level in both tissues. The interplay between ROS levels and survival and the colonization of intracellular bacteria has not been elucidated. Our results indicate that the microbes associated with ticks are dependent on maintaining redox homeostasis while also producing ROS within the tick cells, and they use the ROS as a signaling molecule to regulate the bacterial density [34]. Another possible interpretation is that $\mathrm{Cu} / \mathrm{Zn}$-SOD gene knockdown also affected the enzymatic activity of Mn-SOD (Fig. 3a, b), and the increased generation of superoxide in the salivary glands significantly reduced the total bacterial load. Intriguingly, redox homeostasis is routinely attained by counterbalancing ROS and antioxidants by regulating oxidative stressinduced genes [35], including Cat. The transcriptional expression of Cat was up-regulated slightly in the midgut tissues upon $\mathrm{Cu} / \mathrm{Zn}-\mathrm{SOD}$ knockdown; however, a compensatory role for Cat in increasing the bacterial load cannot be ruled out (Figs. 2a and 4). SOD facilitates the breakdown of $\mathrm{O}^{2-}$ to $\mathrm{H}_{2} \mathrm{O}_{2}$ and molecular oxygen. Mn-SOD (SOD2) from Drosophila mutants show an increased herbicide (Paraquat) sensitivity, elevated endogenous oxidative stress, and reduced longevity [36]. A significant decrease in the total bacterial load upon $\mathrm{Cu} / \mathrm{Zn}-\mathrm{SOD}$ knockdown led us to a follow-up experiment to study the functional role of $\mathrm{Cu} / \mathrm{Zn}-\mathrm{SOD}$ gene depletion on $\mathrm{R}$. parkeri colonization within the tick host.
ROS provide protection against a variety of pathogens by eliciting immune responses in organisms as diverse as mammals and arthropods, including tick responses against $R$. parkeri [4]. Fascinatingly, the interactions between $A$. maculatum and $R$. parkeri determine the ability of these ticks to become colonized by and transmit rickettsial agents. In tick-transmitted infections, the infectious agents manipulate gene expression of the vector host to ensure their colonization and onward transmission to the mammalian host. In the current study, $\mathrm{Cu} / \mathrm{Zn}$-SOD and $\mathrm{Mn}$-SOD transcript levels in the $R$. parkeri-infected ticks showed differential gene expression. Interesting, the transcript level of $M n-S O D$ significantly upregulated $(P<0.05)$ in $R$. parkeriinfected partially blood fed tick tissues and $\mathrm{Cu} / \mathrm{Zn}-\mathrm{SOD}$ transcript level increased only in salivary glands $(P<0.05)$ and remained down-regulated in gut tissues (Fig. 5a). It suggests that tick protects herself from the elevated level of super-oxides generated and released upon rickettsial infection by regulating the expression of superoxide dismutase [37] (Fig. 5a). Interestingly, downregulation of $\mathrm{Cu} / \mathrm{Zn}$ $S O D$ level in $R$. parkeri infected midguts co-related with alleviated $\mathrm{Cu} / \mathrm{Zn}-\mathrm{SOD}$ transcript abundance in partially blood-fed tissues (Fig. 1a). As expected, the $\mathrm{Cu} / \mathrm{Zn}-\mathrm{SOD}$ knockdown up-regulated $M n-S O D$ ( 6.5-fold) expression in the midgut and salivary glands. Surprisingly, the expression levels of Salp25D and SelM were 9-fold and 3fold up-regulated, respectively. It is established that $A$. maculatum eEFSec, I. scapularis glutathione peroxidase (Salp25D), and Dermacentor variabilis SelM offer a survival advantage to $R$. parkeri, B. burgdorferi, and Anaplasma marginale [4, 38, 39].

\section{Conclusions}

This is the first study to show that the silencing of $\mathrm{Cu} /$ $Z n-S O D$ decreases the level of $R$. parkeri colonization in tick tissues. The A. maculatum antioxidants, $M n-S O D$ and $\mathrm{Cu} / \mathrm{Zn}$-SOD play a role in maintaining redox homeostasis within the tick and facilitate the colonization of human pathogenic bacteria, $R$. parkeri. Our findings provide a strong foundation for future research to elucidate the molecular determinants of vector competence. The relationship between ROS, vector competence, and $R$. parkeri-induced antioxidants is undergoing further investigation in our laboratory.

\section{Additional files}

Additional file 1: Figure S1. Evolutionary relationships of taxa based on the SOD amino acid sequence using maximum likelihood method. The evolutionary history was inferred by using the Maximum Likelihood method based on the JTT matrix-based model [15]. The tree is drawn to scale, with the branch lengths measured by the number of substitutions per site. Evolutionary analyses were conducted using MEGA6 software [14]. The sequences were obtained from Amblyomma maculatum, Amblyomma variegatum, Ixodes scapularis, Anopheles gambiae, Mus musculus, Sus scrofa, and Homo sapiens. 
GenBank accession numbers followed by species names are shown in the tree. Figure S2A. Multiple sequence alignments of Cu/Zn-SOD amino acid sequences from different taxa. Regions outlined by red boxes indicate metal-binding sites that are conserved between all of the listed species. The sequences for Cu/Zn-SODs were obtained from Drosophila melanogaster, Apis mellifera, Amblyomma maculatum, Thermostable mutant of Human Cu/Zn SOD, Mus musculus, Equus caballus, Danio rerio, Anopheles gambiae and Culex quinquefasciatus. Figure S2B. Multiple sequence alignments of Mn-SOD amino acid sequences from different taxa. Regions outlined by red boxes indicate metal-binding sites that are conserved between all the listed species. The sequences were obtained from Haliotis discus, Lottia gigantea, Perinereis nuntia, Stegastes partitus, Takifugu rubripes, Struthio camelus, Tauraco erythrolophus, Thamnophis elegans, Sus scrofa, Macaca mulatta, Mus musculus, Xenopus laevis, Amblyomma variegatum, Amblyomma maculatum and lxodes scapularis. Figure S3. The engorged tick weight in dsRNA-SODs injected ticks. The engorged weights of ticks injected with dsLacZ, dsCu/Zn-SOD, dsMn-SOD and dual SODs (dsCu/Zn-SOD and dsMn-SOD) observed at the detachment of ticks from Sheep. There were no significant effects in tick engorged weights with dsRNA-SODs (ANOVA, $\left.F_{(3,52)}=0.6274, P=0.6006\right)$. (DOCX $473 \mathrm{~kb}$ )

\section{Competing interests}

The authors declare that they have no competing interests.

\section{Authors' contributions}

Conceived and designed the experiments: SK. Performed the experiments: GC, KBC, SK. Analyzed the data: GC, KBC, SK. Contributed reagents/materials/ analysis tools: SK. Wrote the paper: GC, KBC, SK. All authors have read and approved the manuscript.

\section{Acknowledgments}

This work was supported by grants from the National Institute of Allergy and Infectious Diseases (award \#Al099919) and the National Institutes of General Medical Sciences (award \#P20GM103476). Gary Crispell is the recipient of the Mississippi-INBRE summer undergraduate research fellowship. These funding bodies played no role in the study design, data collection, analysis, decision to publish, or manuscript preparation.

\section{Received: 12 April 2016 Accepted: 9 May 2016}

\section{Published online: 20 May 2016}

\section{References}

1. Paddock CD, Goddard J. The evolving medical and veterinary importance of the gulf coast tick (Acari: Ixodidae). J Med Entomol. 2015;52:230-52.

2. Fomenko DE, Koc A, Agisheva N, Jacobsen M, Kaya A, Malinouski M, et al. Thiol peroxidases mediate specific genome-wide regulation of gene expression in response to hydrogen peroxide. Proc Natl Acad Sci U S A. 2011;108:2729-34.

3. Karim S, Singh P, Ribeiro JMC. A deep insight into the sialotranscriptome of the gulf coast tick Amblyomma maculatum. PLoS One. 2011;6:e28525.

4. Adamson SW, Browning RE, Budachetri K, Ribeiro JMC, Karim S. Knockdown of selenocysteine-specific elongation factor in Amblyomma maculatum alters the pathogen burden of Rickettsia parkeri with epigenetic control by the Sin3 Histone Deacetylase Corepressor Complex. PLoS One. 2013;8: e82012

5. Adamson S, Browning R, Singh P, Nobles S, Villarreal A, Karim S. Transcriptional activation of antioxidants may compensate for selenop rotein deficiencies in Amblyomma maculatum (Acari: Ixodidae) injected with selK- or selM-dsRNA. Insect Mol Biol. 2014;23(4):497-510.

6. Budachetri K, Karim S. An insight into the functional role of thioredoxin reductase, a selenoprotein, in maintaining normal native microbiota in the Gulf Coast tick (Amblyomma maculatum). Insect Mol Biol. 2015;24:570-81.

7. Hoffmann JA. The immune response of Drosophila. Nature. 2003:426:33-8.

8. Ha E-M, Oh C-T, Ryu J-H, Bae Y-S, Kang S-W, Jang I-H, et al. An antioxidant system required for host protection against gut infection in Drosophila. Dev Cell. 2005;8:125-32.

9. Patrick CD, Hair JA. Laboratory rearing procedures and equipment for multihost ticks (Acarina: Ixodidae). J Med Entomol. 1975;12:389-90.
10. Budachetri K, Browning RE, Adamson SW, Dowd SE, Chao C-C, Ching W-M, et al. An insight into the microbiome of the Amblyomma maculatum (Acari: Ixodidae). J Med Entomol Entomological Society of America. 2014;51:119-29.

11. Thompson JD, Gibson TJ, Higgins DG. Multiple sequence alignment using ClustalW and ClustalX. Curr Protoc Bioinforma. 2002; Chapter 2:Unit 23.

12. Larkin MA, Blackshields G, Brown NP, Chenna R, McGettigan PA, McWilliam $H$, et al. Clustal W and clustal X version 2.0. Bioinformatics. 2007;23:2947-8.

13. Waterhouse AM, Procter JB, Martin DM, Clamp M, Barton GJ. Jalview Version 2 - a multiple sequence alignment editor and analysis workbench. Bioinformatics. 2009;25:1189-91.

14. Tamura K, Stecher G, Peterson D, Filipski A, Kumar S. MEGA6: Molecular evolutionary genetics analysis version 6.0. Mol Biol Evol. 2013;30:2725-9.

15. Jones DT, Taylor WR, Thornton JM. The rapid generation of mutation data matrices from protein sequences. Bioinformatics. 1992:8:275-82.

16. Grimm D, Tilly K, Byram R, Stewart PE, Krum JG, Bueschel DM, et al. Outersurface protein $C$ of the Lyme disease spirochete: a protein induced in ticks for infection of mammals. Proc Natl Acad Sci U S A. 2004;101:3142-7.

17. MORGAN JF, MORTON HJ, PARKER RC. Nutrition of animal cells in tissue culture; initial studies on a synthetic medium. Proc Soc Exp Biol Med. 1950;73:1-8.

18. MORGAN JF, CAMPBELL ME, MORTON HJ. The nutrition of animal tissues cultivated in vitro. I. A survey of natural materials as supplements to synthetic medium 199. J. Natl. Cancer Inst. 1955;16:557-67.

19. Browning R, Adamson SW, Karim S. Choice of a stable set of reference genes for qRT-PCR analysis in Amblyomma maculatum (Acari: Ixodidae). J Med Entomol. 2012:49:1339-46.

20. Kumar D, Budachetri K, Meyers VC, Karim S. Assessment of tick antioxidant responses to exogenous oxidative stressors and insight into the role of catalase in the reproductive fitness of the Gulf Coast tick, Amblyomma maculatum. Insect Mol Biol. 2016;25(3):283-94.

21. Bullard RL, Williams J, Karim S. Temporal gene expression analysis and RNA silencing of single and multiple members of gene family in the lone star tick Amblyomma americanum. PLoS One. 2016;11:0147966.

22. Karim S, Adamson SW, Simpson SJ, Casas J. RNA interference in ticks: A functional genomics tool for the study of physiology. In: Jockusch E, editor. Adv. Insect Physiol. Small RNAs Their Divers. Roles Pract. Uses. I. San Diego: Academic; 2012. p. 119-54

23. Narasimhan S, Rajeevan N, Liu L, Zhao YO, Heisig J, Pan J, et al. Gut microbiota of the tick vector Ixodes scapularis modulate colonization of the Lyme disease spirochete. Cell Host Microbe. 2014;15:58-71.

24. Petersen TN, Brunak S, von Heijne G, Nielsen H. SignalP 4.0: discriminating signal peptides from transmembrane regions. Nat Methods. 2011;8:785-6.

25. Fee JA, Peisach J, Mims WB. Superoxide dismutase. Examination of the metal binding sites by electron spin echo spectroscopy. J Biol Chem. 1981;256:1910-4.

26. Britton L, Fridovich I. Intracellular localization of the superoxide dismutases of Escherichia coli: a reevaluation. J Bacteriol. 1977;131:815-20.

27. Karim S, Ribeiro JMC. An insight into the sialome of the lone star tick, Amblyomma americanum, with a glimpse on its time dependent gene expression. PLoS One. 2015;10, e0131292.

28. Binnington KC. Sequential changes in salivary gland structure during attachment and feeding of the cattle tick, Boophilus microplus. Int J Parasitol. 1978:8:97-115.

29. DeJong RJ, Miller LM, Molina-Cruz A, Gupta L, Kumar S, Barillas-Mury C. Reactive oxygen species detoxification by catalase is a major determinant of fecundity in the mosquito Anopheles gambiae. Proc Natl Acad Sci U S A. 2007;104:2121-6.

30. Orr W, Sohal R. Extension of life-span by overexpression of superoxide dismutase and catalase in Drosophila melanogaster. Science. 1994;263:1128-30.

31. Schriner SE, Linford NJ, Martin GM, Treuting P, Ogburn CE, Emond M, et al. Extension of murine life span by overexpression of catalase targeted to mitochondria. Science. 2005;308:1909-11.

32. Liochev SI, Fridovich I. Superoxide and nitric oxide: consequences of varying rates of production and consumption: a theoretical treatment. Free Radic Biol Med. 2002;33:137-41.

33. Kehrer JP. The Haber-Weiss reaction and mechanisms of toxicity. Toxicology. 2000;149:43-50.

34. Brennan LJ, Haukedal JA, Earle JC, Keddie B, Harris HL. Disruption of redox homeostasis leads to oxidative DNA damage in spermatocytes of Wolbachia-infected Drosophila simulans. Insect Mol Biol. 2012;21:510-20.

35. Dröge W. Free radicals in the physiological control of cell function. Physiol Rev. 2002;82:47-95. 
36. Kirby K, Hu J, Hilliker AJ, Phillips JP. RNA interference-mediated silencing of Sod2 in Drosophila leads to early adult-onset mortality and elevated endogenous oxidative stress. Proc Natl Acad Sci U S A. 2002;99:16162-7.

37. Santucci LA, Gutierrez PL, Silverman DJ. Rickettsia rickettsii induces superoxide radical and superoxide dismutase in human endothelial cells. Infect Immun. 1992;60:5113-8.

38. Narasimhan S, Sukumaran B, Bozdogan U, Thomas V, Liang X, DePonte K, et al. A tick antioxidant facilitates the Lyme disease agent's successful migration from the mammalian host to the arthropod vector. Cell Host Microbe. 2007;2:7-18.

39. Kocan KM, Zivkovic Z, Blouin EF, Naranjo V, Almazán C, Mitra R, et al. Silencing of genes involved in Anaplasma marginale-tick interactions affects the pathogen developmental cycle in Dermacentor variabilis. BMC Dev Biol. 2009;9:42.

Submit your next manuscript to BioMed Central and we will help you at every step:

- We accept pre-submission inquiries

- Our selector tool helps you to find the most relevant journal

- We provide round the clock customer support

- Convenient online submission

- Thorough peer review

- Inclusion in PubMed and all major indexing services

- Maximum visibility for your research

Submit your manuscript at www.biomedcentral.com/submit 